\title{
Clinical effect and standardization of indocyanine green angiography in the laparoscopic colorectal surgery
}

\author{
Gyung Mo Son ${ }^{1,2,3}$, Hong-min Ahn', In Young Lee ${ }^{3}$, Sun Min Lee ${ }^{4}$, Sang-Ho Park ${ }^{5}$, Kwang-Ryul Baek \\ ${ }^{1}$ Department of Surgery, Pusan National University Yangsan Hospital, Yangsan, Korea \\ ${ }^{2}$ Research Institute for Convergence of Biomedical Science and Technology, Pusan National University Yangsan Hospital, Yangsan, Korea \\ ${ }_{3}^{3}$ Medical Research Center, Pusan National University School of Medicine, Yangsan, Korea \\ ${ }^{4}$ Department of Laboratory Medicine, Pusan National University Yangsan Hospital, Yangsan, Korea \\ ${ }^{5}$ Department of Electronic Engineering, Pusan National University, Busan, Korea
}

\begin{abstract}
Anastomotic complications occur after $5 \%$ to $20 \%$ of operations for rectosigmoid colon cancer. The intestinal perfusion status at the anastomotic site is an important modifiable risk factor, and surgeons should carefully evaluate and optimize the perfusion at the intended site of anastomosis. Indocyanine green (ICG) angiography is a simple noninvasive perfusion assessment modality. The use of ICG angiography is rapidly spreading in the field of colorectal surgery. However, there is debate on its contribution to reducing anastomotic complications. In this review, we discuss the clinical utility and the standardization of ICG angiography. ICG angiography can unequivocally reveal unfavorable perfusion zones and provide quantitative parameters to predict the risk of hypoperfusion-related anastomotic complications. Many studies have demonstrated the clinical utility of ICG angiography for reducing anastomotic complications. Recently, two multicenter randomized clinical trials reported that ICG angiography did not significantly reduce the incidence of anastomotic leakage. Most previous studies have been small-scale single-center studies, and there is no standardized ICG angiography protocol to date. Additionally, ICG angiography evaluations have mostly relied on surgeons' subjective judgment. For these reasons, it is necessary to establish a standardized ICG angiography protocol and develop a quantitative analysis protocol for the objective assessment. In conclusion, ICG angiography could be useful for detecting poorly perfused colorectal segments to prevent anastomotic leakage after colorectal surgery. An optimized and standardized ICG angiography protocol should be established to improve the reliability of perfusion assessments. In the future, artificial intelligence-based quantitative analyses could be used to easily assess colonic perfusion status.
\end{abstract}

Keywords: Indocyanine green, Angiography, Quantitative light-induced fluorescence, Anastomotic leak, Colorectal surgery

This is an Open Access article distributed under the terms of the Creative Commons Attribution Non-Commercial License (http:/ creativecommons.org/licenses/by-nc/4.0/) which permits unrestricted non-commercial use, distribution, and reproduction in any medium, provided the original work is properly cited.

Received August 16, 2021

Revised September 6, 2021

Accepted September 8, 2021

Corresponding author

Gyung Mo Son

Department of Surgery, Pusan

National University Yangsan

Hospital, 20 Geumo-ro, Mulgeum-

eup, Yangsan 50612, Korea

Tel: +82-55-360-2124

Fax: +82-55-360-2154

E-mail: skm1711@pusan.ac.kr

ORCID:

https://orcid.org/0000-0002-8861-6293
Copyright (C) The Korean Society of Endo scopic and Laparoscopic Surgeons.

\section{INTRODUCTION}

Colorectal cancer is one of the commonest gastrointestinal tumors. The rectosigmoid colon is the most common location for colon cancer. Anastomotic complications occur after approxi- mately $5 \%$ to $20 \%$ of anterior resections for rectosigmoid colon cancer [1]. Risk factors associated with anastomotic complications vary considerably, but they include the tension of anastomosis, perfusion status, distance of the anastomosis from the anal verge, preoperative chemoradiation, smoking, advanced stage, difficult 
total mesorectal excision, male patient, stapler characteristics, anemia, and nutrition [2]. Most of these variables are unmodifiable patient-related risk factors. However, there are some modifiable risk factors that are gradually becoming possible to overcome with technological advances. Intestinal perfusion status has become a modifiable risk factor, and surgeons can evaluate and select anastomosis sites with optimal perfusion to perform safe colorectal anastomoses [3].

Ligation of the inferior mesenteric artery (IMA) has been considered an essential step for oncological dissection of the rectosigmoid colon. Several studies have reported that IMA ligation may cause hypoperfusion of the left-sided colon [4,5]. Almost half of anastomotic leaks are suspected to be associated with hypoperfusion. Subjective assessments have been used to evaluate blood flow in the gastrointestinal tract. This might have limited diagnostic accuracy dependent on the surgeon's experience. Indocyanine green (ICG) angiography is a simple, noninvasive method that is carried out using a near-infrared (NIR) camera system. Currently, ICG angiography is rapidly gaining adoption in colorectal surgery. The clinical utility of ICG angiography for reducing anastomotic complications is under scientific investigation through several multicenter randomized clinical trials [6,7]. The expectations and interests of ICG angiography among surgeons are continually increasing. However, since ICG angiography has been carried out with various protocols, standardization is required $[8,9]$. In this review, we discuss the clinical utility and standardization of ICG angiography for colonic perfusion assessment.

\section{MAIN SUBJECTS}

\section{Prolonged debate about inferior mesenteric artery ligation and hypoperfusion}

IMA ligation in rectosigmoid colon cancer surgery is commonly performed for sufficient D3 lymph node dissection and tensionfree anastomosis through sufficient proximal colon lengthening. However, it may increase the risk of damage to the autonomic nerve plexus of the IMA root and ischemia of the left-sided colon. To overcome this risk, some surgeons perform low ligation of the IMA to preserve the left colic artery [10]. This procedure requires a high level of surgical expertise for vascular branch dissection. However, D3 lymph node dissection cannot be performed as an en bloc resection, and controversy remains over oncological safety for low IMA ligation even though these two procedures have not been shown to significantly affect oncologic outcomes [11]. The IMA could be a mesenteric anchoring point in the cases of short sigmoid colons and advanced rectosigmoid colon cancer. Therefore, high ligation is inevitably selected to obtain sufficient redundancy of the proximal colonic segment.
When performing colorectal surgery, anastomotic complications are the most difficult hurdles faced by surgeons and patients. The large intestine has a different blood flow supply system on the right and left-sided colon. The marginal arterial arcade of the right and left-sided colon might be connected near the splenic flexure, forming a collateral circulation between the superior mesenteric artery (SMA) and the IMA. The lower rectum is supplied with blood flow by the middle and lower rectal arteries branching from the internal iliac artery. Therefore, even when IMA ligation accompanies anterior resection, blood flow in the left colon can be maintained by the SMA. However, an anatomical cadaver study reported that in $20 \%$ of individuals, linkage of the marginal arcade forming the collateral circulation might be insufficient [12].

Hypoperfusion of the left side of the colon is a well-known phenomenon associated with IMA ligation. We reported on marginal arterial pressures measured through mesenteric artery cannulation, particularly looking at changes in colonic mesenteric artery pressure before and after the IMA ligation; we measured marginal arterial pressure before and after IMA clamping. In more than $10 \%$ of rectosigmoid cancer patients, marginal arterial pressure was significantly decreased by more than $30 \%$ [4]. Therefore, colonic hypoperfusion might be a rare but real event, potentially leading to ischemic colitis in some patients with poor collateral circulation. To prevent hypoperfusion-related anastomotic complications, this $10 \%$ of high-risk patients with ischemic changes must be identified.

Experienced surgeons have traditionally practiced observing intestinal color, arterial pulsation, and colonic peristalsis, or bleeding at the cutting edge of the mesenteric artery. These subjective evaluations could be considered good clinical assessments by experienced surgeons. However, the accuracy and reliability of subjective evaluations could have a wide spectrum depending on surgical experience [13]. Accordingly, an objective and quantitative technique is needed to improve the accuracy and reliability of bowel perfusion status evaluations. Such an evaluation method should be noninvasive and easy to apply, and the results could be interpreted intuitively in real time by the surgeon. Additionally, the diagnostic technique used should be able to be safely applied to the patient for measuring colonic perfusion [14].

There are a variety of methods for evaluating blood flow. Arterial pressure can be measured through arterial cannulation via connections to blood pressure monitoring equipment in the operating room. [4,15]. Color Doppler ultrasonography is a safe, noninvasive procedure, which has the advantage of allowing repeated and safe evaluation of perfusion status in real time. However, there are technical limitations for evaluating very small blood vessels within 1-2 mm of the colonic wall [16]. Computed tomography (CT) angiography can be used for assessing the mesenteric arterial structure. CT resolution has been gradually 
improving and now permits the evaluation of the arterial circuit using three-dimensional reconstruction. However, CT angiography has a limited resolution for visualization of the collateral marginal artery connection with very small vascular diameters of less than $1 \mathrm{~mm}$ at the splenic flexure [4].

Therefore, a simple and noninvasive assessment method is needed for obtaining high-resolution images in real time for daily practice in the era of minimally invasive surgery.

\section{Indocyanine green angiography and anastomotic leakage}

ICG is a fluorescent agent that was approved by the U.S. Food and Drug Administration in 1956. To date, it is the only fluorescent substance that can be administered to humans for clinical practice. Although ICG-related side effects are rare, allergic reactions may occur, particularly when a patient has a history of CT contrast allergy. ICG solutions contain a trace amount of iodine, which is a component of CT contrast solution [17]. Therefore, it is necessary to review a patient's allergy history before performing ICG angiography; it is also important to monitor for allergic reactions during the examination.

ICG becomes fluorescent at around $800 \mathrm{~nm}$, which is within the NIR wavelength range. NIR spectroscopy was developed to detect ICG fluorescence, and it is possible to use this technology to visualize ICG in blood samples. This function is mainly used to evaluate the reserve liver function before liver transplantation or liver resection [18]. However, the equipment used for this investigation is still so large that the collected venous blood should be measured in a special laboratory for ICG concentration measurement. Since ICG binds to proteins, such as albumin and globulins in the blood, extravasation does not occur easily, which is useful for evaluating blood flow. ICG was first applied to cardiac function evaluation and ophthalmoscopic angiography. In the surgical field, a handheld NIR camera was developed to evaluate blood flow by detecting fluorescence from intravascular ICG. Recently, a laparoscopic NIR camera has been developed, making ICG angiography available for use in minimally invasive surgery [19]. With ICG angiography, a surgeon can directly observe the intestinal perfusion status with a fluorescence image. Changes in the fluorescence intensity (FI) are viewed along the path of blood flow to the target organ. Fluorescence images can be expressed in green, cyan, or blue on a black-and-white background, depending on the NIR camera system [20]. A fluorescence image can also be expressed as an overlay on the same image of a white light view.

ICG angiography can be used to unequivocally reveal unfavorable perfusion zones and obtain quantitative parameters to predict the risk of complications associated with hypoperfusion. Transection line changes have been reported in 5\% to $20 \%$ of patients with unfavorable perfusion segments identified by ICG angiography. At our institution, the anastomotic complication rate reached nearly $10 \%$ before we instituted ICG angiography, and it rapidly decreased to $1 \%$ to $2 \%$ after we started evaluating colonic perfusion [3]. Many studies have demonstrated the clinical utility of ICG angiography for reducing anastomotic complications (Table 1) [3,5-7,13,14,19,21-41].

However, two multicenter randomized clinical trials reported that ICG angiography did not significantly reduce the incidence of anastomotic leakage [6,7]. Nevertheless, a meta-analysis determined that there were significantly fewer anastomotic complications in patients who underwent ICG angiography in the included studies (Table 2) [42-49]. One of the reasons for these contradictory results is that quantitative analysis and optimal standardized protocols are still lacking for ICG angiography. Most of the existing evidence has been obtained from smallscale, single-center studies, and the ICG angiography protocol has not been standardized. Additionally, the evaluation of ICG angiography has mostly relied on the subjective judgment of the surgeon, and few studies have applied quantitative analyses. For these reasons, it is necessary to establish a standardized ICG angiography protocol and develop a quantitative analysis protocol for objectively assessing FI.

\section{Standardization of indocyanine green angiography protocol}

To date, various NIR devices and different angiography protocols have been applied at different institutions. Therefore, to compare and analyze image data from multiple institutions, we need to establish a standardized ICG angiography protocol to improve efficacy and accuracy. Additionally, qualitative perfusion parameters could improve the accuracy of a surgeon's subjective judgment. The ability to detect changes in FI is a breakthrough in fluorescence imaging that allows tissue perfusion to be easily visualized [3]. However, in ICG angiography, FI is greatly affected by the external environment. Other quantitative parameters based on FI also depend on external conditions. For example, one study found the maximal FI level and increasing slope to vary significantly, influenced by various conditional factors, including perfusion status [9]. FI scale can be changed because it is quite sensitive to external environmental conditions.

We previously applied various external conditions to obtain the best fluorescence images for several years. From these experiences, we found the optimal external environmental conditions for maximizing FI [9]. The distance between the camera and tissue was one of the most important determinants of brightness. In current ICG cameras for laparoscopy, the brightness changes sharply according to the distance when it is closer than 4 to $5 \mathrm{~cm}$; when it is further than 4 to $5 \mathrm{~cm}$, the brightness becomes relatively stable, which reduces the quality of evaluation of FI chang- 


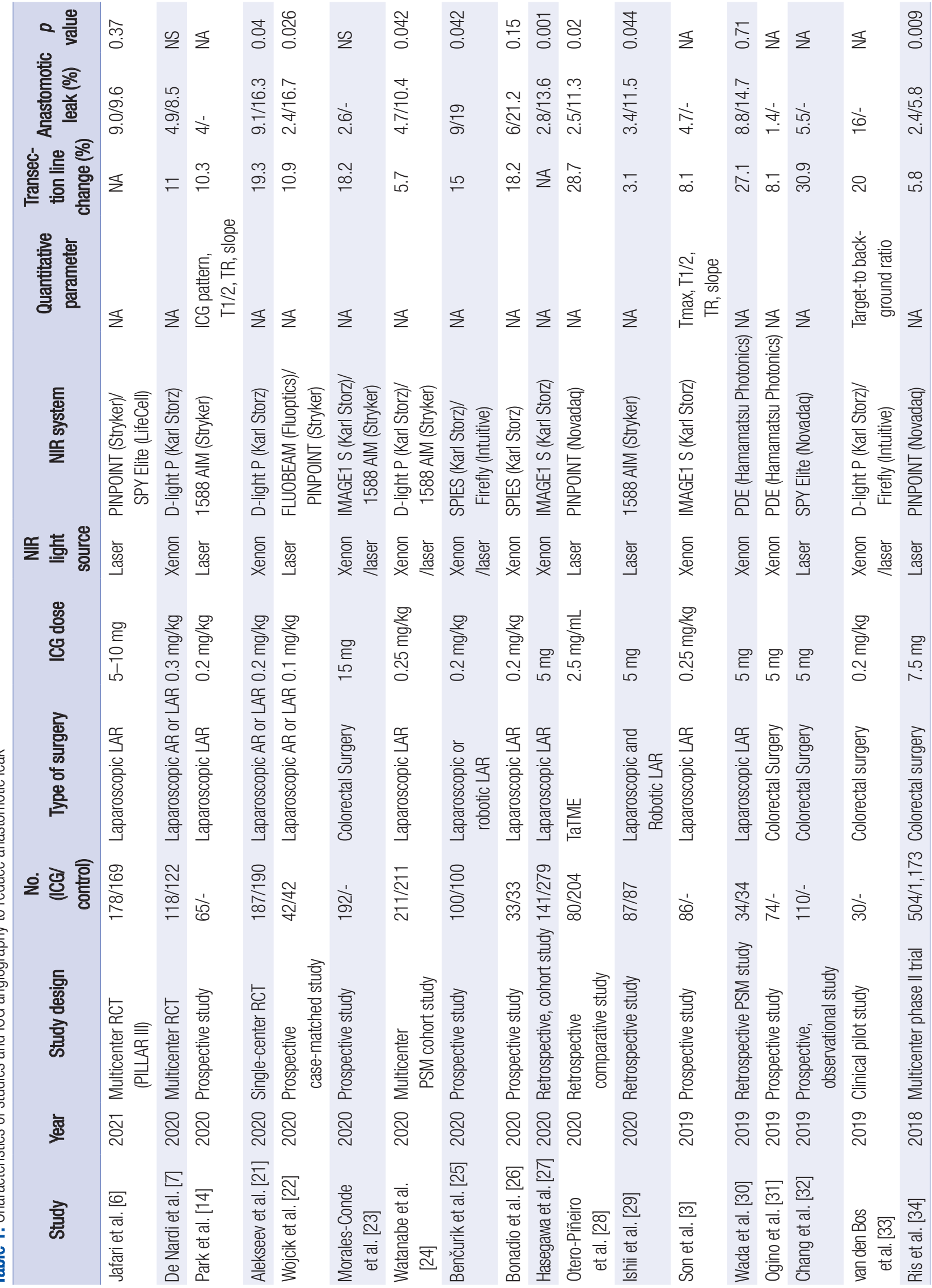




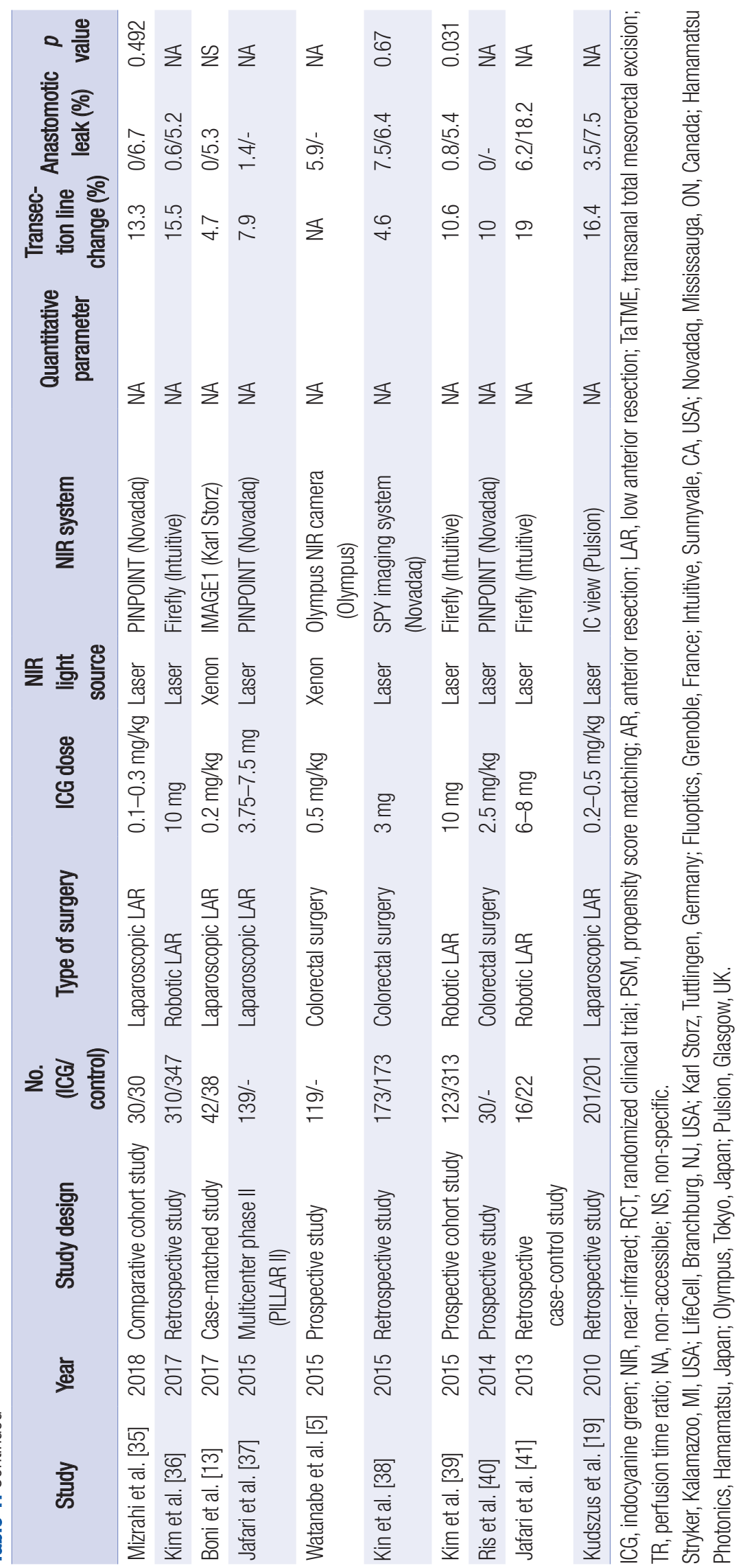


Table 2. Meta-analysis for clinical effects of ICG angiography to reduce anastomotic leak

\begin{tabular}{|lccllllll}
\hline \multicolumn{1}{c}{ Study } & Year & $\begin{array}{c}\text { No. of } \\
\text { enrolled } \\
\text { study }\end{array}$ & $\begin{array}{c}\text { No. } \\
\text { (ICG/control) }\end{array}$ & \multicolumn{1}{c}{ Type of surgery } & $\begin{array}{c}\text { Anasto- } \\
\text { motic } \\
\text { leak (\%) }\end{array}$ & $\begin{array}{c}\text { Odds } \\
\text { ratio }\end{array}$ & $95 \% \mathrm{Cl}$ & $p$ value \\
\hline Zhang and Che [42] & 2021 & 29 & $2,354 / 3,522$ & AR and LAR & $3.2 / 9.2$ & 0.39 & $0.30-0.50$ & $<0.00001$ \\
\hline Arezzo et al. [43] & 2020 & 9 & $862 / 468$ & Colorectal surgery & $4.2 / 11.3$ & 0.34 & $0.22-0.53$ & $<0.001$ \\
\hline Liu et al. [44] & 2020 & 13 & $1,806 / 2,231$ & Colorectal surgery & $3.8 / 7.8$ & 0.4 & $0.33-0.59$ & $<0.00001$ \\
\hline Lin et al. [45] & 2020 & 11 & $1,364 / 1,773$ & Colorectal surgery & $2.9 / 30.8$ & 0.31 & $0.21-0.44$ & $<0.0001$ \\
\hline Chan et al. [46] & 2020 & 20 & $2,220 / 3,278$ & Colorectal surgery & $3.7 / 8.6$ & 0.46 & $0.31-0.62$ & $<0.00001$ \\
\hline Rausa et al. [47] & 2019 & 11 & $555 / 1,768$ & Colorectal surgery & $3.6 / 6.0$ & 0.44 & $0.14-0.87$ & $<0.05$ \\
\hline Shen et al. [48] & 2018 & 4 & $569 / 608$ & Laparoscopic and robotic LAR & $1.8 / 6.4$ & 0.27 & $0.13-0.53$ & $<0.0002$ \\
\hline Blanco-Colino et al. [49] & 2018 & 5 & $555 / 747$ & Colorectal surgery & $1.1 / 6.1$ & 0.51 & $0.23-1.13$ & 0.10 \\
\hline
\end{tabular}

ICG, indocyanine green; AR, anterior resection; LAR, low anterior resection; $\mathrm{Cl}$, confidence interval.

es. Therefore, with laparoscopic ICG cameras, it is expected that the optimal fluorescence image can be obtained by measuring fluorescence at a distance of 4 to $5 \mathrm{~cm}$. Additionally, it is helpful to obtain a fluorescence image of good quality by performing ICG angiography in the abdominal cavity or in as dark an environment as possible by turning off all room lights when capturing images from outside the abdominal cavity. Moreover, using a laser rather than a xenon lamp as a fluorescence emission source is advantageous for obtaining a fluorescence image of higher resolution and quality. Even with ICG laparoscopy using xenon lamps, the ICG-specific image mode can improve the quality of fluorescence images through image processing methods, such as red color inversion. Under these premises, when the conditions for fluorescence imaging are satisfied, we will be able to evaluate the colonic perfusion status using quantitative parameters, such as ascending slope or maximum FI.

FI and persisting duration are affected by the dilution concentration and dose of the intravenous ICG injection. In our previous study for ICG angiography, a typical dilution concentration of $2.5 \mathrm{mg} / \mathrm{mL}$ was prepared, and an intravenous dose of $0.25 \mathrm{mg} /$ $\mathrm{kg}$ was applied according to the patient's weight. In early ICG cameras using xenon lamps, blue fluorescence was visualized, so there was a resolution limitation in which the difference between the background and the blue color tone was not clear, so the appropriate ICG dose was set at $0.25 \mathrm{mg} / \mathrm{kg}$. The initial ICG study of our research team also applied a dose of 0.2 to $0.25 \mathrm{mg} /$ $\mathrm{kg}$ using a laparoscopic NIR camera with a xenon lamp. As the fluorescence emission source for the laparoscopic camera is a laser, and with improvements in fluorescence imaging resolution, research on the optimal ICG dosage is required for repetitive ICG angiography. Optimized ICG angiography should be able to show a definite FI change. If necessary, repeated ICG angiography could be performed without disturbances from remnant fluorescence in the tissues. Therefore, the optimal ICG dose should also be rapidly cleared by the liver, facilitating repeat ICG angiography. We conducted a study to find the optimal ICG capacity for quantitative fluorescence analysis using a laser NIR camera and compared the fluorescence images between standard $(0.2 \mathrm{mg} / \mathrm{kg})$ and experimental $(0.01-0.05 \mathrm{mg} / \mathrm{kg})$ dosage groups. In our recent study, sufficient quantitative perfusion evaluation was possible even with a low dose of $0.02 \mathrm{mg} / \mathrm{kg}$, which is ten times lower than the conventional dose of $0.2 \mathrm{mg} / \mathrm{kg}$ (unpublished data).

\section{Quantitative perfusion analysis}

After 10 to 30 seconds of intravenous ICG injection, fluorescence begins to appear in the colon wall. It is possible to evaluate perfusion status by changing FI to ensure that the perfusion is well preserved to the planned transection line.

A time-fluorescence graph could be displayed by measuring changes in FI, allowing evaluation of various quantitative parameters. In the measurement of FI, the basic quantitative factors are the maximal level of $\mathrm{FI}\left(\mathrm{F}_{\max }\right)$ and the interval to $\mathrm{FI}_{\max }\left(\mathrm{T}_{\max }\right)$. Time scales include latency time (the initial fluorescence onset time), the interval to half of $\mathrm{FI}_{\max }\left(\mathrm{T}_{1 / 2 \max }\right)$, and perfusion time ratio (TR). The ascending slope can reflect arterial flow conditions and can be calculated considering both FI and time scale (Fig. 1). Since the perfusion status of the small intestine can be used as a criterion for a good FI level, the perfusion status can also be evaluated by calculating the FI ratio of the small intestine and colon [30].

Among these variables, many studies have explored $\mathrm{T}_{1 / 2 \max }$. In cases of insufficient blood flow, $T_{1 / 2 \max }$ delays have been reported in a number of digestive organs, such as the small intestine and large intestine. We found that $\mathrm{T}_{1 / 2 \max }$ and TR can reflect blood flow conditions well [3]. In particular, $\mathrm{T}_{1 / 2 \max }$ has the highest sensitivity for predicting anastomotic complications, and TR is 
analyzed as a variable with high specificity. Therefore, we proposed a step-by-step flow chart to improve diagnostic accuracy. At first, $\mathrm{T}_{1 / 2 \max }$ can be used to screen for patients at risk of insufficient perfusion; second, TR can be used to predict the risk of anastomotic complications [3].

Based on the tissue perfusion status information, the surgeon will be able to perform safer colonic anastomoses by clearly identifying colonic segments with excellent blood flow. In particular, such quantitative perfusion evaluation would be of great help to a novice surgeon who lacks experience in subjective evaluation of tissue perfusion [50]. ICG angiography allows the surgeon to

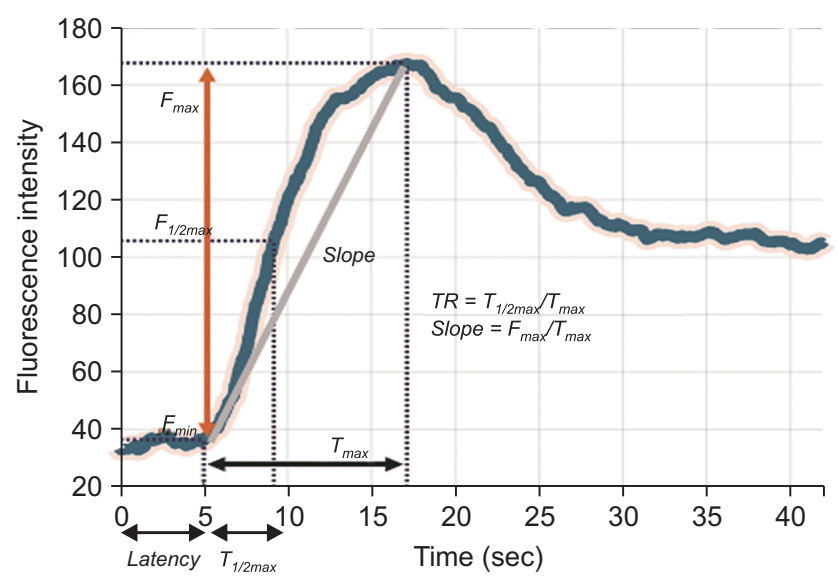

Fig. 1. Quantitative parameters based on the time-fluorescence of indocyanine green angiography. This graph could be displayed by measuring changes in the fluorescence intensity (FI), allowing evaluation of various quantitative parameters. In the measurement of $\mathrm{Fl}$, the basic quantitative factors are the maximal level of $\mathrm{FI}\left(\mathrm{F}_{\max }\right)$ and the interval to $\mathrm{FI}_{\max }\left(\mathrm{T}_{\max }\right)$. Time scales include latency time (the initial fluorescence onset time), the interval to half of $\mathrm{FI}_{\max }\left(\mathrm{T}_{1 / 2 \max }\right)$, and perfusion time ratio (TR). The ascending slope can reflect arterial flow conditions and can be calculated considering both $\mathrm{Fl}$ and time scale. Adapted from Ahn et al. [9], according to the Creative Commons License. intuitively estimate tissue perfusion in real time. Eventually, ICG angiography may facilitate a reduction in anastomotic complications associated with poor colonic perfusion.

For this, a safe and reliable ICG angiography protocol must be established. Additionally, it is necessary to develop and verify accurate analytical indicators for predicting the risk of complications and quantitatively evaluating the state of tissue microperfusion in real time.

Experienced surgeons can empirically assess tissue perfusion without ICG angiography, but surgical beginners do not. However, manual evaluation by novice surgeons is subject to error. Accurate evaluation requires many years of practice to obtain sufficient experience. On the other hand, artificial intelligence (AI) can evaluate tissue perfusion with the highest accuracy via learning using numerous ICG angiography videos. The processed information can then be used to supplement the surgeon's judgment.

In the fluorescence graph used for quantitative analysis, a nonideal pattern may be observed due to the various blood flow pathways of the vascular structures and collateral circulation. In such a case, the quantitative parameter would be incorrectly recorded. This could be a hidden pitfall potentially leading to interpretation errors in perfusion evaluation. Additionally, environmental factors of ICG angiography such as NIR illuminance and lighting outside the shooting distance can affect the perfusion evaluation. Quantitative analysis has this limitation, but AI can be used to overcome it. AI, which has learned both patterns and various environmental factors, has superior analysis performance and stability relative to quantitative analysis. Real-time AI analysis can be used to quickly evaluate the state of the tissue microcirculation so that analysis results can be checked right in the operating room. The results can be expressed visually through a color map overlying the surgical field (Fig. 2). When the limited data for machine learning is solved in the future, it is expected that this will play an important role as a reliable assistant in the operating room [14].
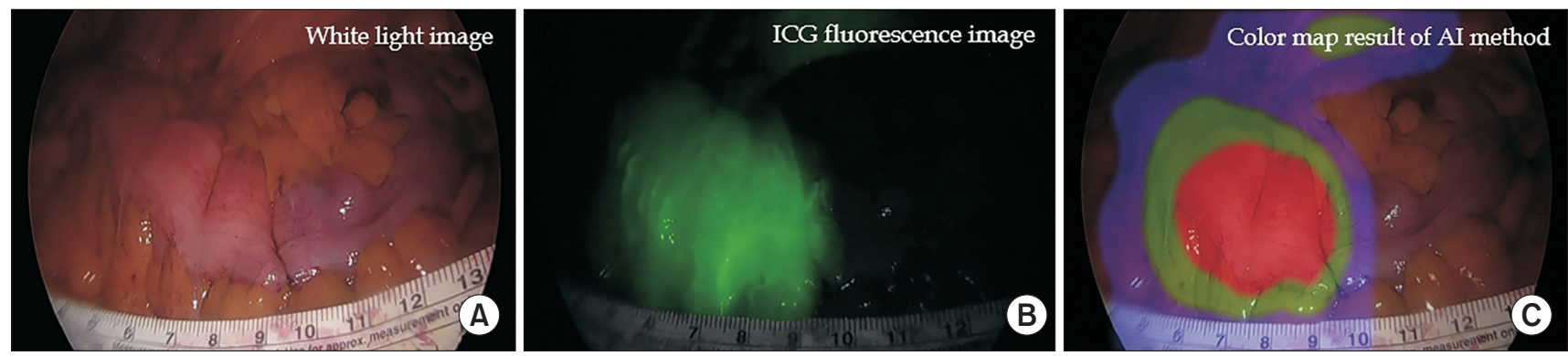

Fig. 2. Colonic perfusion images. (A) White light image, (B) indocyanine green (ICG) fluorescence image, and (C) color map result of artificial intelligence (Al) analysis of ICG pattern. Real-time Al-based quantitative analysis can be used to evaluate the tissue microcirculation and express visually through a color map overlying the surgical field. The visual information can help the surgeons assess the perfusion status during laparoscopic surgery. Adapted from Park et al. [14], according to the Creative Commons License. 


\section{PERSPECTIVES}

Currently, various fluorescence imaging devices, fluorescent drugs, hyperspectral image, and laser speckle contrast imaging systems are being rapidly developed. ICG fluorescence images can be obtained more delicately through the second window using a wavelength of $1,000 \mathrm{~nm}$ or more. This can increase the resolution of ICG fluorescence images and improve transmittance to achieve deeper penetration of fluorescence [51]. In addition to ICG, various fluorescent materials are currently being developed for clinical applications. Fluorescence imaging of the urinary system, as well as the biliary tract, can be performed using the different metabolic pathways applicable to fluorescent drugs [52]. Additionally, window fluorescence drugs have been developed for tissue-specific fluorescence imaging in the laboratory.

Aside from ICG angiography, tissue perfusion status and oxygen saturation levels can be determined using hyperspectral imaging or laser speckle contrast imaging. This new imaging technique will be able to noninvasively evaluate in vivo physiological states using the physical properties of light without fluorescent drugs, such as ICG. A tissue-specific imaging technology has also been developed for reflecting the physiological characteristics of tissues [53].

We are fortunate to be able to stand in the middle of a revolutionary era of fluorescence surgical technology and surgical navigation systems. Currently, surgeons have a new imaging device at their disposal, with which they can easily and safely apply ICG angiography in the operating room. By evaluating tissue microcirculation in real time, tissues with poor blood flow can be accurately identified. The risk factors of hypoperfusion can be predicted intraoperatively, and postoperative complications can be prevented. These advanced imaging technologies will improve the safety of colorectal surgery, which will eventually bring medical and economic benefits to doctors, patients, and health care systems. We believe that this seemingly small step in the operating room could prove to be a giant leap toward preventing anastomotic complications for colorectal cancer patients.

\section{CONCLUSION}

ICG angiography could be useful for detecting poorly perfused colonic segments and preventing anastomotic leakage after colorectal surgery. An optimal standardized ICG angiography protocol should be established to facilitate the implementation of reliable multicenter clinical trials to confirm the clinical benefits of ICG angiography. In the future, we envision AI-based quantitative analysis software being used to assess colonic perfusion during laparoscopic or robotic colorectal surgery.

\section{NOTES}

\section{Authors' contributions}

Conceptualization: GMS

Formal Analysis: GMS, HMA, SHP

Investigation: GMS

Methodology: GMS, SHP, IYL, KRB

Writing-Original Draft: GMS, HMA, SML, SHP

Writing-Review \& Editing: GMS

All authors read and approved the final manuscript.

\section{Conflict of interest}

All authors have no conflicts of interest to declare.

\section{Funding/support}

This study was supported by a 2021 research grant from Pusan National University Yangsan Hospital.

\section{ORCID}

Gyung Mo Son, https://orcid.org/0000-0002-8861-6293

Hong-min Ahn, https://orcid.org/0000-0001-9963-2021

In Young Lee, https://orcid.org/0000-0001-5954-6188

Sun Min Lee, https://orcid.org/0000-0002-2896-3365

Sang-Ho Park, https://orcid.org/0000-0001-6380-301X

Kwang-Ryul Baek, https://orcid.org/0000-0002-2928-2043

\section{REFERENCES}

1. Yeung TM. Fluorescence imaging in colorectal surgery. Surg Endosc 2021;35:4956-4963.

2. Park JS, Choi GS, Kim SH, et al. Multicenter analysis of risk factors for anastomotic leakage after laparoscopic rectal cancer excision: the Korean laparoscopic colorectal surgery study group. Ann Surg 2013;257:665-671.

3. Son GM, Kwon MS, Kim Y, Kim J, Kim SH, Lee JW. Quantitative analysis of colon perfusion pattern using indocyanine green (ICG) angiography in laparoscopic colorectal surgery. Surg Endosc 2019;33:1640-1649.

4. Son GM, Kim TU, Park BS, et al. Colonic hypoperfusion following ligation of the inferior mesenteric artery in rectosigmoid colon cancer patients. Ann Surg Treat Res 2019;97:74-82.

5. Watanabe J, Ota M, Suwa Y, et al. Evaluation of the intestinal blood flow near the rectosigmoid junction using the indocyanine green fluorescence method in a colorectal cancer surgery. Int J Colorectal Dis 2015;30:329-335

6. Jafari MD, Pigazzi A, McLemore EC, et al. Perfusion Assessment 
in Left-Sided/Low Anterior Resection (PILLAR III): a randomized, controlled, parallel, multicenter study assessing perfusion outcomes with PINPOINT near-infrared fluorescence imaging in low anterior resection. Dis Colon Rectum 2021;64:995-1002.

7. De Nardi P, Elmore U, Maggi G, et al. Intraoperative angiography with indocyanine green to assess anastomosis perfusion in patients undergoing laparoscopic colorectal resection: results of a multicenter randomized controlled trial. Surg Endosc 2020;34:53-60.

8. Baiocchi GL, Guercioni G, Vettoretto N, et al. ICG fluorescence imaging in colorectal surgery: a snapshot from the ICRAL study group. BMC Surg 2021;21:190.

9. Ahn HM, Son GM, Lee IY, Park SH, Kim NS, Baek KR. Optimization of indocyanine green angiography for colon perfusion during laparoscopic colorectal surgery. Colorectal Dis 2021;23:1848-1859.

10. Fujii S, Ishibe A, Ota M, et al. Short-term and long-term results of a randomized study comparing high tie and low tie inferior mesenteric artery ligation in laparoscopic rectal anterior resection: subanalysis of the HTLT (High tie vs. low tie) study. Surg Endosc 2019;33:1100-1110.

11. Matsuda K, Yokoyama S, Hotta T, et al. Oncological outcomes following rectal cancer surgery with high or low ligation of the inferior mesenteric artery. Gastrointest Tumors 2017;4:45-52.

12. Brandt LJ, Feuerstadt P, Longstreth GF, Boley SJ; American College of Gastroenterology. ACG clinical guideline: epidemiology, risk factors, patterns of presentation, diagnosis, and management of colon ischemia (CI). Am J Gastroenterol 2015;110:18-44.

13. Boni L, Fingerhut A, Marzorati A, Rausei S, Dionigi G, Cassinotti E. Indocyanine green fluorescence angiography during laparoscopic low anterior resection: results of a case-matched study. Surg Endosc 2017;31:1836-1840.

14. Park SH, Park HM, Baek KR, Ahn HM, Lee IY, Son GM. Artificial intelligence based real-time microcirculation analysis system for laparoscopic colorectal surgery. World J Gastroenterol 2020;26:69456962.

15. Guo Y, Wang D, He L, et al. Marginal artery stump pressure in left colic artery-preserving rectal cancer surgery: a clinical trial. ANZ J Surg 2017;87:576-581.

16. Seike K, Koda K, Saito N, et al. Laser Doppler assessment of the influence of division at the root of the inferior mesenteric artery on anastomotic blood flow in rectosigmoid cancer surgery. Int J Colorectal Dis 2007;22:689-697.

17. Kim M, Lee S, Park JC, et al. Anaphylactic shock after indocyanine green video angiography during cerebrovascular surgery. World Neurosurg 2020;133:74-79.

18. Zheng J, Xie W, Huang Y, Zhu Y, Jiang L. The technique of 3D reconstruction combining with biochemistry to build an equivalent formula of indocyanine green (ICG) clearance test to assess the liver reserve function. BMC Surg 2020;20:283.

19. Kudszus S, Roesel C, Schachtrupp A, Höer JJ. Intraoperative laser fluorescence angiography in colorectal surgery: a noninvasive analysis to reduce the rate of anastomotic leakage. Langenbecks Arch Surg
2010;395:1025-1030.

20. Ahn HM, Son GM, Lee IY, et al. Optimal ICG dosage of preoperative colonoscopic tattooing for fluorescence-guided laparoscopic colorectal surgery. Surg Endosc 2021 Feb 26 [Epub]. https://doi.org/10.1007/ s00464-021-08382-5.

21. Alekseev M, Rybakov E, Shelygin Y, Chernyshov S, Zarodnyuk I. A study investigating the perfusion of colorectal anastomoses using fluorescence angiography: results of the FLAG randomized trial. Colorectal Dis 2020;22:1147-1153.

22. Wojcik M, Doussot A, Manfredelli S, et al. Intra-operative fluorescence angiography is reproducible and reduces the rate of anastomotic leak after colorectal resection for cancer: a prospective casematched study. Colorectal Dis 2020;22:1263-1270.

23. Morales-Conde S, Alarcón I, Yang T, et al. Fluorescence angiography with indocyanine green (ICG) to evaluate anastomosis in colorectal surgery: where does it have more value? Surg Endosc 2020;34:38973907.

24. Watanabe J, Ishibe A, Suwa Y, et al. Indocyanine green fluorescence imaging to reduce the risk of anastomotic leakage in laparoscopic low anterior resection for rectal cancer: a propensity score-matched cohort study. Surg Endosc 2020;34:202-208.

25. Benčurik V, Škrovina M, Martínek L, et al. Intraoperative fluorescence angiography and risk factors of anastomotic leakage in miniinvasive low rectal resections. Surg Endosc 2021;35:5015-5023.

26. Bonadio L, Iacuzzo C, Cosola D, et al. Indocyanine green-enhanced fluorangiography (ICGf) in laparoscopic extraperitoneal rectal cancer resection. Updates Surg 2020;72:477-482.

27. Hasegawa H, Tsukada Y, Wakabayashi M, et al. Impact of intraoperative indocyanine green fluorescence angiography on anastomotic leakage after laparoscopic sphincter-sparing surgery for malignant rectal tumors. Int J Colorectal Dis 2020;35:471-480.

28. Otero-Piñeiro AM, de Lacy FB, Van Laarhoven JJ, et al. The impact of fluorescence angiography on anastomotic leak rate following transanal total mesorectal excision for rectal cancer: a comparative study. Surg Endosc 2021;35:754-762.

29. Ishii M, Hamabe A, Okita K, et al. Efficacy of indocyanine green fluorescence angiography in preventing anastomotic leakage after laparoscopic colorectal cancer surgery. Int J Colorectal Dis 2020;35:269275.

30. Wada T, Kawada K, Hoshino N, et al. The effects of intraoperative ICG fluorescence angiography in laparoscopic low anterior resection: a propensity score-matched study. Int J Clin Oncol 2019;24:394-402.

31. Ogino T, Hata T, Kawada J, et al. The risk factor of anastomotic hypoperfusion in colorectal surgery. J Surg Res 2019;244:265-271.

32. Chang YK, Foo CC, Yip J, et al. The impact of indocyanine-green fluorescence angiogram on colorectal resection. Surgeon 2019;17:270276.

33. van den Bos J, Jongen ACHM, Melenhorst J, Breukink SO, Lenaerts K, Schols RM, Bouvy ND, Stassen LP. Near-infrared fluorescence image-guidance in anastomotic colorectal cancer surgery and its rela- 
tion to serum markers of anastomotic leakage: a clinical pilot study. Surg Endosc 2019;33:3766-3774.

34. Ris F, Liot E, Buchs NC, et al. Multicentre phase II trial of near-infrared imaging in elective colorectal surgery. Br J Surg 2018;105:13591367.

35. Mizrahi I, Abu-Gazala M, Rickles AS, et al. Indocyanine green fluorescence angiography during low anterior resection for low rectal cancer: results of a comparative cohort study. Tech Coloproctol 2018;22:535-540.

36. Kim JC, Lee JL, Park SH. Interpretative guidelines and possible indications for indocyanine green fluorescence imaging in robot-assisted sphincter-saving operations. Dis Colon Rectum 2017;60:376-384.

37. Jafari MD, Wexner SD, Martz JE, et al. Perfusion assessment in laparoscopic left-sided/anterior resection (PILLAR II): a multi-institutional study. J Am Coll Surg 2015;220:82-92.

38. Kin C, Vo H, Welton L, Welton M. Equivocal effect of intraoperative fluorescence angiography on colorectal anastomotic leaks. Dis Colon Rectum 2015;58:582-587.

39. Kim JC, Lee JL, Yoon YS, Alotaibi AM, Kim J. Utility of indocyaninegreen fluorescent imaging during robot-assisted sphincter-saving surgery on rectal cancer patients. Int J Med Robot 2016;12:710-717.

40. Ris F, Hompes R, Cunningham C, et al. Near-infrared (NIR) perfusion angiography in minimally invasive colorectal surgery. Surg Endosc 2014;28:2221-2226.

41. Jafari MD, Lee KH, Halabi WJ, et al. The use of indocyanine green fluorescence to assess anastomotic perfusion during robotic assisted laparoscopic rectal surgery. Surg Endosc 2013;27:3003-3008.

42. Zhang W, Che X. Effect of indocyanine green fluorescence angiography on preventing anastomotic leakage after colorectal surgery: a meta-analysis. Surg Today 2021;51:1415-1428.

43. Arezzo A, Bonino MA, Ris F, et al. Intraoperative use of fluorescence with indocyanine green reduces anastomotic leak rates in rectal cancer surgery: an individual participant data analysis. Surg Endosc 2020;34:4281-4290.

44. Liu D, Liang L, Liu L, Zhu Z. Does intraoperative indocyanine green fluorescence angiography decrease the incidence of anastomotic leakage in colorectal surgery?: a systematic review and meta-analysis. Int J Colorectal Dis 2021;36:57-66.

45. Lin J, Zheng B, Lin S, Chen Z, Chen S. The efficacy of intraoperative ICG fluorescence angiography on anastomotic leak after resection for colorectal cancer: a meta-analysis. Int J Colorectal Dis 2021;36:27-39.

46. Chan DK, Lee SK, Ang JJ. Indocyanine green fluorescence angiography decreases the risk of colorectal anastomotic leakage: systematic review and meta-analysis. Surgery 2020;168:1128-1137.

47. Rausa E, Zappa MA, Kelly ME, et al. A standardized use of intraoperative anastomotic testing in colorectal surgery in the new millennium: is technology taking over?: a systematic review and network meta-analysis. Tech Coloproctol 2019;23:625-631.

48. Shen R, Zhang Y, Wang T. Indocyanine green fluorescence angiography and the incidence of anastomotic leak after colorectal resection for colorectal cancer: a meta-analysis. Dis Colon Rectum 2018;61:1228-1234.

49. Blanco-Colino R, Espin-Basany E. Intraoperative use of ICG fluorescence imaging to reduce the risk of anastomotic leakage in colorectal surgery: a systematic review and meta-analysis. Tech Coloproctol 2018;22:15-23.

50. Kim DH, Son GM, Kwon MS, Baek SH, Park BS, Kim HS. Educational benefits of intraoperative indocyanine green angiography for surgical beginners during laparoscopic colorectal surgery. J Minim Invasive Surg 2018;21:25-30.

51. Ma Z, Zhang M, Yue J, et al. Near-infrared IIb fluorescence imaging of vascular regeneration with dynamic tissue perfusion measurement and high spatial resolution. Adv Funct Mater 2018;28:1803417.

52. Fushiki H, Yoshikawa T, Matsuda T, Sato T, Suwa A. Preclinical development and validation of ASP5354: a near-infrared fluorescent agent for intraoperative ureter visualization. Mol Imaging Biol 2021 May 11 [Epub]. https://doi.org/10.1007/s11307-021-01613-0.

53. Jansen-Winkeln B, Holfert N, Köhler H, et al. Determination of the transection margin during colorectal resection with hyperspectral imaging (HSI). Int J Colorectal Dis 2019;34:731-739. 